\title{
Appearance Control using Projection with Model Predictive Control
}

\author{
Toshiyuki Amano, Hirokazu Kato \\ Graduate School of Information Science, Nara Institute of Science and Technology \\ \{amano,kato\}@is.naist.jp
}

\begin{abstract}
The unified technique for the irradiance correction and appearance enhancement for the real scene is proposed in this paper. The proposed method employed MPC algorithm for the projector camera system and enabled arbitrary appearance control such like photo retouching software in the real world. In the experiment, the appearance control of saturation enhancement, color removal, phase control, edge enhancement, image blur, makes unique brightness and the other enhancements for the real scene are shown.
\end{abstract}

\section{Introduction}

The projector camera system has abilities of optical compensation, appearance enhancement, and it enables us to change visual appearance in the real world. The optical compensation is not only for the irradiance correction on the textured screen but also to control the appearance of colored and textured object has been proposed as an application. In contrast of compensation, projector camera system makes possible to enhance the original appearance of the object [6]. With the overlay projection on the object with the original appearance, we can get contrast enhanced the appearance in the real world $[3,1]$. As well as contrast enhancement, we can apply saturation enhancement, tone mapping, highlight clipping, etc with the image processing during in projection process. The application of this technique is not limited for pictures and other publications, and it is possible to apply to the optical microscopy [2]. These techniques are possible to apply for 3D object easily with employ of the per-pixel mapping between projector and camera coordinates. With the coaxial optics that employed beam-splitter to combine projection and capturing, it is possible to apply dynamical scene without recalculation of per-pixel mapping [5]. For the dynamical adaptation, some feedback methods have been proposed for optical compensation [5] and appearance enhance- ment [1] independently. These purposes are different, but both of techniques are based on same optical control technique and same equipments. However, these techniques are not structured as a general methodology based on the reliable control theory. This paper proposes unified methodology for dynamical appearance control that employed Model Prediction Control (MPC) algorithm.

\section{Projector Camera Feedback}

\subsection{Model Predictive Control}

The Dynamic Adaptation algorithm [5] can be explained as one of MPC algorithm [4] that commonly used in the process industries such as chemical plants and oil refineries since 1980s. This control method is including a model for control object, and the manipulation value is decided by using of this model. Its idea is simple and reasonable way when we have known the system response. By using of model prediction $y_{M}(t)$ that includes input $u(t)$ and measurement $y(t)$, estimation of output $y_{P}(t+1)$ is given by

$$
y_{P}(t+1)=y_{M}(t+1)+\operatorname{Error}(t)
$$

where

$$
\operatorname{Error}(t)=y(t)-y_{M}(t)
$$

for considering of estimation error. In the MPC algorithm, reference trajectory

$$
y_{R}(t+1)=\alpha(t) y(t)+(1-\alpha(t)) r(t+1)
$$

is used for control stability against modeling error. Since $y_{P}(t+1)=y_{R}(t+1)$, we get control input for step $t+1$, where $\alpha(t)$ and $r(t)$ are tuning parameter and desired value for step $t$. The Dynamic Adaptation is corresponding with this algorithm at the condition of $\alpha(t)=0$ and $\operatorname{Error}(t)=0$. Fortunately, projector camera feedback is stable without these terms in case of irradiance compensation since control error is decreasing step by step. However, we have to consider these 


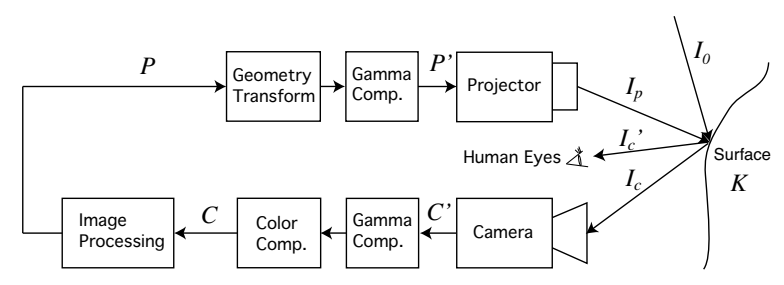

Figure 1. Iterative Projection

terms in the appearance enhancement since its error is critical for the appearance enhancement.

\subsection{Negative Feedback for the Enhancement}

Amano et. al. proposed appearance enhancement technique with projector camera feedback [1]. The diagram is shown in figure 1 . In this diagram, all property have 3 color components of $R, G$ and $B$ channel. Now we think about for a single pixel relation. The environment light $I_{0} \in \mathcal{R}^{3}$ and projection light $I_{p} \in \mathcal{R}^{3}$ is mixed and reflected on the surface $K \in \mathcal{R}^{3 \times 3}$. This reflection

$$
I_{c}=K\left(I_{p}+I_{0}\right)
$$

is captured by camera and the system get an image

$$
C=M C_{c}^{\prime \gamma}, C^{\prime} \propto I_{c}
$$

after the gamma and color compensation. Regarding of lambert reflection, we can expect an irradiance that observed by user $I_{c}^{\prime} \approx I_{c}$. Where $M$ is the color mixing matrix between camera and projector color spaces. After the image processing, the system projects compensation light $P$ with correct scaled irradiance on to the same point with the captured pixel from projector by using of the geometrical transformation and gamma compensation. The remarkable point is the projection light $I_{P}$ never diverge with proper image processing includes gain control. However, it is not easy to reach desired appearance since to estimate final result is decided with many times iteration of this feedback. Moreover, this feedback model is not robust since this model is not include projection error nor capture error. In other words, the problems are this system has no reference image for desired appearance neither not employed negative feedback that considered control error. To solve these problems, I propose a new feedback approach that shown in fig. 2. The estimated physical surface reflectance is written as

$$
\hat{K}=\operatorname{diag}\left\{C \cdot /\left\{\left(C_{\text {full }}-C_{0}\right) \odot P+C_{0}\right\}\right\}
$$

by using projection pattern. Where $C_{\text {full }}$ and $C_{0}$ are images those are captured images under maximum

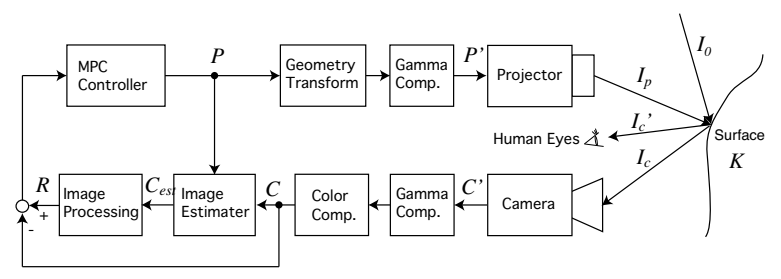
Figure 2. Negative Feedback with MPC
controller

power ( complete white image ) and minimum power ( black image ) projections, ./ and $\odot$ are meaning a component wise division and multiplication respectively. Note, however, those non-linear response features of $C$ and $P$ are compensated with the module of "Gamma Comp.". Based on $\hat{K}$, the true appearance that under the white light projection is given by

$$
C_{e s t}=\hat{K} C_{w h i t e},
$$

where $C_{\text {white }}=(1,1,1)^{T}$. Since this true appearance, we can get reference image for negative feedback loop after the arbitrary image processing.

\subsection{MPC for Appearance Enhancement}

To apply MPC algorithm, I use projector camera response model

$$
C_{M}(t+1)=\hat{K}(t)\left\{\left(C_{\text {full }}-C_{0}\right) \odot P(t+1)+C_{0}\right\},
$$

where $P(t), C(t) \in([0,1],[0,1],[0,1])^{T}$ are normalized projection pattern and captured image at the step $t$. Image prediction that considered model error is written by

$$
C_{P}(t+1)=C_{M}(t+1)+\operatorname{Error}(t)
$$

where

$$
\operatorname{Error}(t)=C(t)-C_{M}(t) .
$$

As for the reference trajectory,

$$
C_{R}(t+1)=\alpha C(t)+(1-\alpha) R(t+1)
$$

is used. That employed constant tuning parameter since projector camera response is stay flat for $t \geq 0$. Where $R(t+1)$ is a reference image that is given by the part of image processing based on true appearance. From the control law $C_{P}(t+1)=C_{R}(t+1)$, we get manipulating value

$$
\begin{aligned}
& P(t+1) \\
& \approx \hat{K}(t)^{-1}(1-\alpha)\{R(t+1)-C(t)\} \\
& \quad \cdot /\left(C_{\text {full }}-C_{0}\right)+P(t)
\end{aligned}
$$


with an approximation of $\hat{K}(t) \approx \hat{K}(t-1)$. This approximation is employed to avoid near zero division when $\hat{K}(t-1) \approx 0$ that leads unstable control. This approximation is sufferable since image prediction eq. (9) is including modeling error.

\section{Experimental Results}

The system for experiment is composed with VGA resolution IEEE1394 camera (Bayer filter format, 30fps.) and XGA resolution PC projector (3LCD, $60 \mathrm{~Hz}$.). Pixel mapping between coordinates of camera and projector is calibrated with general calibration method employed gray code projection. The scene shown in figure 3(a) is used for evaluation. This scene is taken under white light projection. The saturation and contrast of appearance can be controlled with an iterative projection [1], but it is not stable and it reached reddish enhanced result with high gained parameters shown in figure 3(b). On the contrary, a stable saturation enhancement result by proposed method with comparable parameter is shown in the figure 3(c).

The advantage is not limited to its stability and accuracy but also its versatility since it employed a reference image in the feedback process and it yields various appearance control other than color saturation enhancement shown in fig. 4(b). To apply color to gray scale conversion for the image processing of $C_{e s t}$, the system can remove color from the real scene such like fig. 4(c). In this result, the color of the scene is completely removed as like as gray scale publications. The color removal process is an opposite process of color saturation enhancement, but it cannot be done without reference image because it is hard to estimate parameter in case of iterative projection. The system can control the color of scene with color phase convert for the image processing not only saturation (Fig. 4(d)). The appearance control technique used projection is bound by the optical theory. Therefore, it is impossible to make appearance darker than its original appearance, and cannot convert its color to opposite for pure colored object. However, proposed method has many abilities for the appearance control and it is possible to enhance edge, make blur and make brightness uniquely. Figure 4(e) shows the results of edge enhancement and edge blur. From left to right, the picture shows the edge enhancement, original appearance, and blur result. The L-G filter and Gaussian Blur are used for the edge enhancement and blur respectively. To use of illuminance unification in the image processing, the system can make illuminance of the real scene uniquely such as figure 4(f). In this result, the illumination of the scene is not completely flat because the projection power is saturated at the black color regions. However, it is interesting because the shade is completely removed. This effect might be useful for the segmentation or image analysis based on color phase. The irradiance correction is also possible just using constant gray image for the reference input. The other appearance control can be applied with various image processing(g) (i). The process speed includes image processing is reached 13fps with $2.4 \mathrm{GHz}$ cpu PC(Mac Book Pro Core2Duo 2.4GHz).

\section{Conclusions}

In this paper, the unified methodology for dynamical appearance control that includes irradiance correction and appearance enhancement was proposed. This methodology enables arbitrary appearance control as if it were a photo-retouch software in the real scene with projector camera feedback. In the experiment, saturation enhancement, color removal, color phase control, edge enhancement, make blur, make unique brightness and the other enhancements were shown. I believe those capabilities are not limited to the amusement application, but also helpful for photographer, publisher, conservator of the oil paintings, and visually impaired persons as well.

\section{Acknowledgments}

The authors would like to express my gratitude to Prof. Oliver Bimber at Johannes Kepler University and Anselm Grundhöfer at Bauhaus University for the valuable discussions. This research was partially supported by KAKENHI for Young Scientists (B), 21700137.

\section{References}

[1] T. Amano and H. Kato. Real world dynamic appearance enhancement with procam feedback. In Proc. of the 5 th International Workshop on PROCAMS, pages 1-2, 2008.

[2] O. Bimber, A. Grundhöfer, D. Kurz, S. Thiele, F. Häntsch, T. Amano, and D. Klöck. Projected light microscopy. In SIGGRAPH 2009: Talks, pages 1-1, 2009.

[3] O. Bimber and D. Iwai. Superimposing dynamic range. In ACM SIGGRAPH Asia 2008, pages 1-8, 2008.

[4] J. E. N.-R. Eduardo F. Camacho, Carlos Bordons. Model predictive control. Springer, Berlin, 1999.

[5] K. Fujii, M. D. Grossberg, and S. K. Nayar. A projectorcamera system with real-time photometric adaptation for dynamic environments. In Proc. of the Computer Vision and Pattern Recognition - Volume 2, page 1180, 2005.

[6] M. D. Grossberg, H. Peri, S. K. Nayar, and P. N. Belhumeur. Making one object look like another: Controlling appearance using a projector-camera system. In Proc. of the Computer Vision and Pattern Recognition, volume 1, pages 452-459, 2004. 


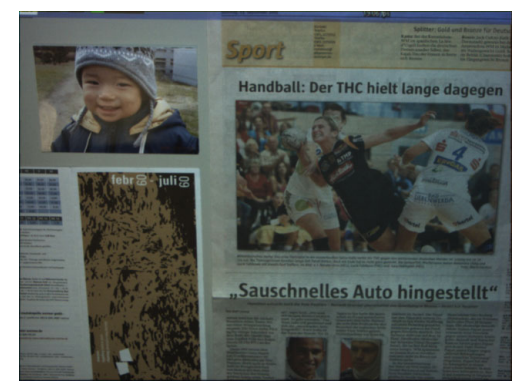

(a) The Orignal Appearance

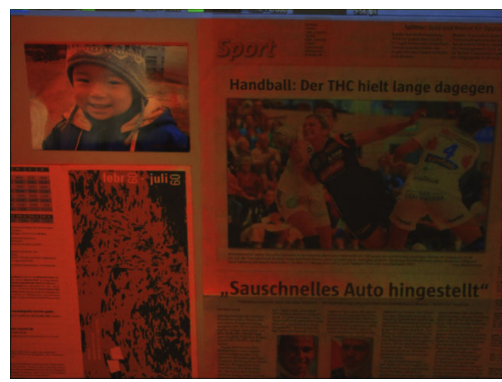

(b) Iterative Projection [1]

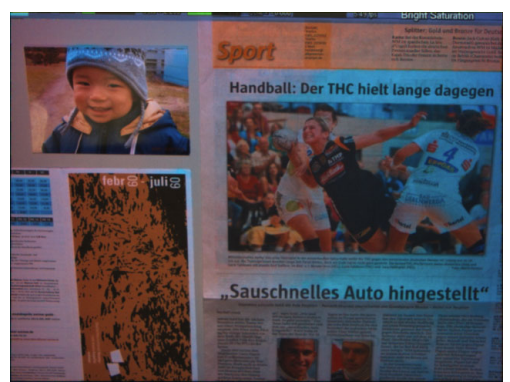

(c) MPC with Modulation Estimation

Figure 3. Color Saturation Enhancement.

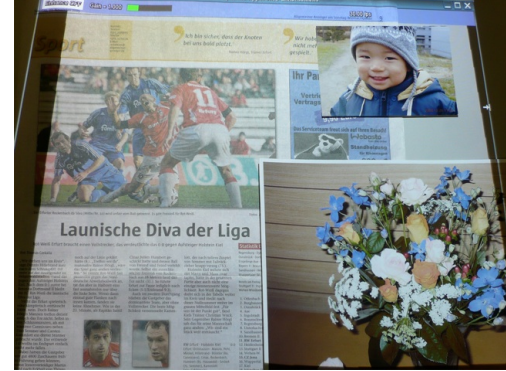

(a) Original Appearance

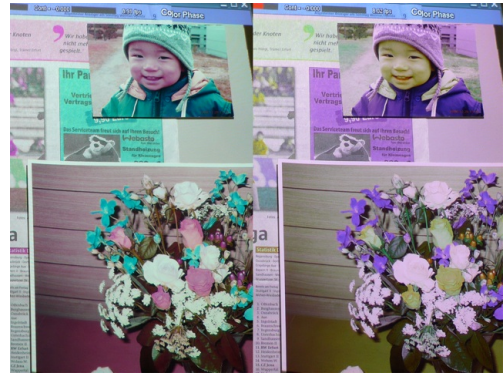

(d) Color Phase Control

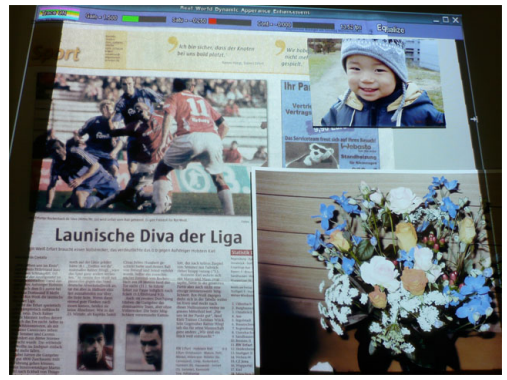

(g) Contrast Boost

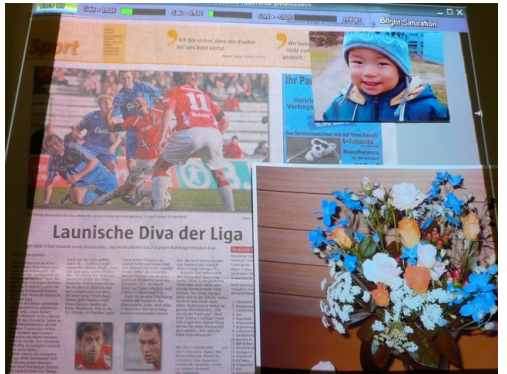

(b) Saturation Enhencement

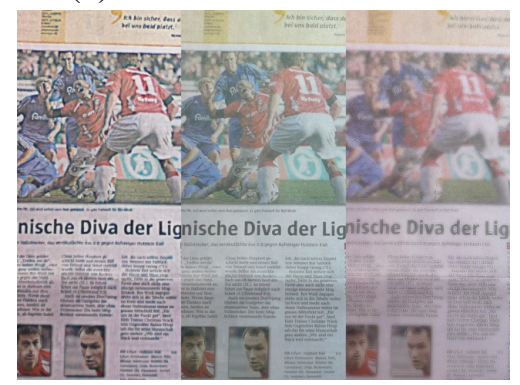

(e) Edge Enhance and Blur

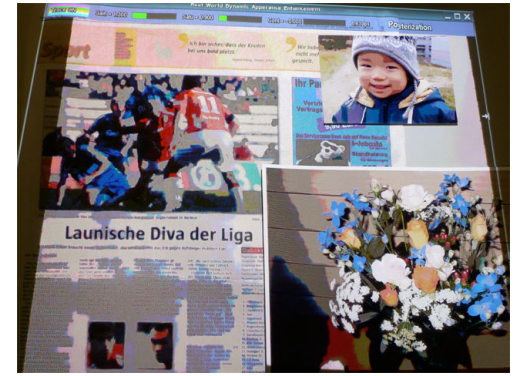

(h) Posterization

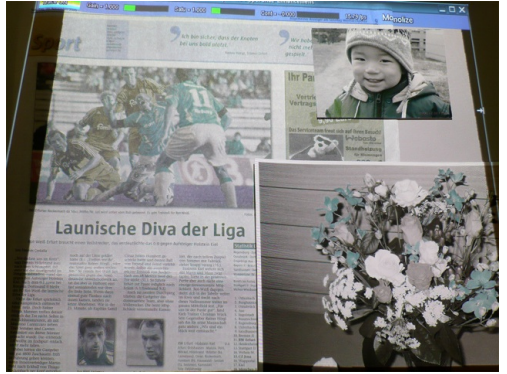

(c) Color Removal

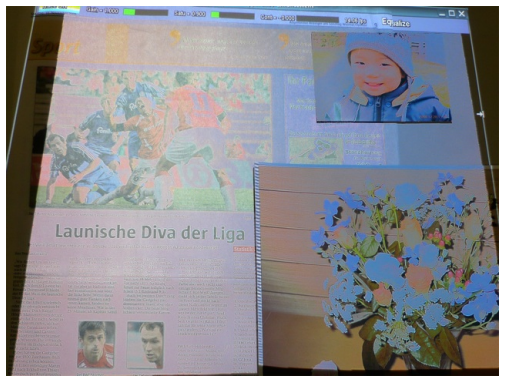

(f) Unique Brightness

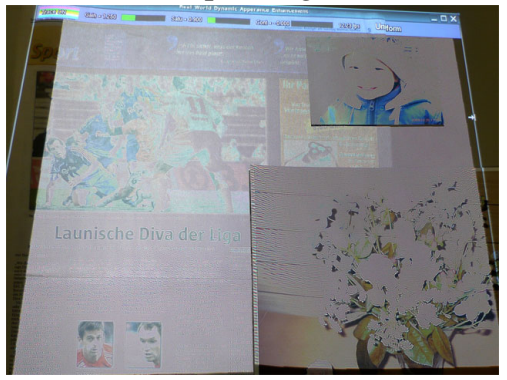

(i) Optical Hiding

Figure 4. Various Appearance Control. 